\title{
A importância do nutricionista na equipe multidisciplinar para o acompanhamento da doença de Huntington
}

\section{The importance of the nutritionist in the multidisciplinary team to follow up Huntington's disease}

\author{
Ziane da Conceição das Mercês ${ }^{1}$ \\ ${ }^{1}$ Especialização em Nutrição e exercício aplicado a prevenção e ao tratamento de doenças. Nutricionista responsável técnico da prefeitura \\ municipal de Aveiro. Pará, Brasil. https://orcid.org/0000-0003-0646-2022 E-mail: zianemerces@gmail.com *Autor para correspondência
}

\author{
Palavras-chave \\ Coreia de Huntington \\ Movimentos involuntários \\ Dietoterapia
}

\begin{abstract}
A doença é uma desordem neurológica degenerativa progressiva causada por uma expansão da repetição dos trinucleotídeos citosina, adenina e guanina presente no cromossomo 4. Objetivo: Demonstrar a importância do nutricionista na equipe multidisciplinar para o acompanhamento do paciente com doença de Huntington. Metodologia: Pesquisa fundamentada em revisão bibliográfica, com analise em estudos publicados nos últimos dez anos, pesquisados em bases fidedignas como: Scielo, Bireme, Pubmed, Lilacs, Google acadêmico. Resultados: Através de uma minuciosa análise em referenciais teóricos, foram selecionados 70 artigos científicos sobre a patologia neurodegenerativa Huntington, desse montante, apenas 47 artigos serviram de base para a composição deste estudo, os 23 estudos restantes estavam fora do período estabelecido, entretanto, serviram para o conhecimento histórico da doença. Todas as pesquisas selecionadas foram lidas na integra e continham em sua composição dados que corroboraram positivamente, justificando sua utilização. Conclusão: Os acometidos apresentam necessidades de acompanhamento domiciliar ou hospitalar, o que seria de grande importância que esse acompanhamento fosse feito por uma equipe multidisciplinar da saúde composta por diversos profissionais entre esses destacando o nutricionista e sua importante atuação.
\end{abstract}

Keywords

Huntington's korea Involuntary movements Diet therapy

\begin{abstract}
The disease is a progressive degenerative neurological disorder caused by an expansion of repeat cytosine, adenine and guanine trinucleotides present on chromosome 4. Objective: To demonstrate the importance of the nutritionist in the multidisciplinary team for monitoring Huntington's disease patients. Methodology: Research based on bibliographic review, with analysis in studies published in the last ten years, researched in reliable bases such as: Scielo, Bireme, Pubmed, Lilacs, Google scholar. Results: Through a thorough analysis of theoretical frameworks, 70 scientific articles were selected on the Huntington neurodegenerative pathology, of which, only 47 articles served as the basis for the composition of this study, the remaining 23 studies were outside the established period, however, they served for historical knowledge of the disease. All selected surveys were read in full and contained in their composition data that corroborated positively, justifying their use. Conclusion: Those affected have needs for home or hospital monitoring, which would be of great importance if a multidisciplinary health team composed of several professionals, highlighting the nutritionist and his important performance, did this monitoring.
\end{abstract}


completa, causando o desenvolvimento da doença de Huntington (SPLITZ, 2010).

A etiologia de Huntington caracteriza-se pela deterioração motora progressiva, perturbações psiquiátricas, humor irritável, disfagia, desnutrição severa, colapso de memória, declínio cognitivo (GONÇALVES, 2013), movimentos involuntários desordenados, arrítmicos, aleatórios, sequências de movimentos que causam uma aparência de postura inquieta, provavelmente causados pela disfunção neuronal ou pela morte das células neurológicas, particularmente no striatum (COTRIM; ALCÂNTARA, 2016). A inconstância motora é a característica observada no exame físico neurológico, tratando-se de uma doença genética (CUNHA; LOPES, 2016), o Huntington exige uma investigação precisa a nível molecular, sondagem das características clínicas, para possível diagnóstico definitivo (MERCÊS et al., 2018).

Segundo a descrição de Cunha e Lopes (2016, p. 111).

O diagnóstico da doença de Huntington baseia-se nos sinais e sintomas de coreia num indivíduo com história familiar confirmada de doença. Atualmente, a possibilidade de pesquisa da alteração genética no gene da proteína huntingtina permite confirmar analiticamente a doença. O estabelecimento do diagnóstico definitivo é feito na especialidade de neurologia, após exclusão de causas secundárias (atrás referidas), achados imagiológicos sugestivos e genotipagem.

Na alimentação, muitas funções do sistema nervoso são exigidas; desta forma, algum transtorno em uma dessas funções pode afetar a capacidade de um indivíduo manter a nutrição adequada e, consequentemente, resultar na incapacidade de cumprir as demandas metabólicas (LIMA; OLIVEIRA, 2016 p.113).

Os problemas nutricionais prevalentes nas demências são a perda de peso, fator de risco para a desnutrição, bem como a desidratação. Tais condições tornam os indivíduos suscetíveis a infecções, agravamento da doença e diminuição acentuada na ingestão de alimentos e consequentemente de calorias e nutrientes (MENDES et al., 2016 p. 503).

Diante do exposto sob a referida patologia o presente estudo questiona: Será que pessoas diagnosticadas com doença de Huntington estão devidamente assistidas por uma equipe multidisciplinar composta por nutricionista e outros profissionais? A presença de um nutricionista na equipe multidisciplinar para o acompanhamento dos pacientes com doença de Huntington, pode contribuir no tratamento, diminuindo os agravos causados pelos sintomas da desnutrição severa, podendo corroborar com um prognóstico satisfatório.

Objetivou-se demonstrar a importância do nutricionista na equipe multidisciplinar para o acompanhamento do paciente com doença de Huntington.

\section{MATERIAL E MÉTODOS}

Pesquisa fundamentada em revisão bibliográfica, com analise em estudos publicados nos últimos dez anos, pesquisados em bases fidedignas como: Scielo, Bireme, Pubmed, Lilacs, Google acadêmico. Para o agrupamento de dados por intermédio das bases eletrônicas, foram utilizados os descritores, doença de Huntington, coreia de Huntington, síndrome coreica, publicados nos últimos 10 anos (2010 2019).

\section{RESULTADOS E DISCUSSÃO}

Através de uma minuciosa análise em referenciais teóricos, foram selecionados 70 artigos científicos sobre a patologia neurodegenerativa Huntington, desse montante, apenas 47 artigos serviram de base para a composição deste estudo, os 23 estudos restantes estavam fora do período estabelecido, entretanto, serviram para o conhecimento histórico da doença. Todas as pesquisas selecionadas foram lidas na integra e continham em sua composição dados que corroboraram positivamente, justificando sua utilização.

As doenças neurodegenerativas acometem milhares de pessoas, um grupo de patologias que cursam com disfunção e posterior morte de neurônios tanto no sistema nervoso central como no periférico (GLITER et al., 2017). Incluindo nesse crescente grupo de patologias neurológicas as doenças de Alzheimer, Parkinson, Huntington, Esclerose Lateral Amiotrófica, (MACEDO et al., 2019).

Historicamente, no século XIV, na França, juntamente com a Peste Negra foi transcrita uma epidemia denominada de coreomania (mania dançante) que teve como característica movimentos arrítmicos (SILVA, 2017). Posteriormente, a coreomania foi chamada de Dança ou Coreia de São Vito, por ter características epidêmicas de coreia histérica. Em 1872, George Huntington, expôs a coreia (derivando da palavra grega xopeía para se referir à dança) com características hereditárias, manifestações motoras, cognitivas e psiquiátricas (PAULA, 2015; SPITZ, 2010), desta forma o termo Coreia foi sucedido por "Doença de Huntington" (GONÇALVES, 2013).

A Doença de Huntington afeta pessoas por todo o mundo, de todas as raças/cor, mas em especial aos 
caucasianos, tendo uma apresentação parecida entre homens e mulheres, e a média de acometimento no mundo é de 6,5 pessoas por 100 mil habitantes (CARVALHO, 2018). No Reino Unido um estudo detectou um aumento considerável nas pessoas acometidas, sendo que a prevalência de $\mathrm{DH}$ mais que dobrou em duas décadas de 5,4 em 1990, para 12,3 em 2010 por 100 mil habitantes (EVANS et al., 2013).

Lago Maracaibo na Venezuela denota uma elevada prevalências, segundo Gonçalves (2013), cerca de 7000 pessoas são diagnosticada com Huntington para cada milhão de habitantes. Os estudos indicam que a prevalência da DH varia muito de acordo com a distribuição geográfica e características da população (XU; WU, 2015). No Brasil não há estudos epidemiológicos que indiquem a epidemiologia da doença, mas estima-se que a incidência seja de 5 a 10 acometidos para cada 100 mil habitantes (FERRAZ et al., 2013).

A herança da doença Huntington é autossômica dominante, ou seja, pode afetar tanto homens quanto mulheres na mesma probabilidade. Pais que apresentam o gene multado transfere aos filhos $50 \%$ de probabilidade de desenvolver a doença (NANCE et al., 2011).

As alterações genéticas transcritas na doença de Huntington estão diretamente ligadas ao cromossomo 4, apresentando-se como uma patologia hereditária e autossômica dominante, tendo como principais características os movimentos involuntários, mudanças comportamentais, disfagia resultando em dificuldade para alimentar-se, sendo todos os sintomas citados são provocados pela morte progressiva das células neurológicas (CAIRES et al., 2018). Pereira (2015, p. 18), reforça em seus argumentos que o Huntington apresenta uma desordem neurológica degenerativa progressiva causada pela expansão das trincas dos nucleotídeos Citosina, Adenina e Guanina presentes no gene IT15 no braço curto do cromossomo 4. Descrições semelhantes sobre as alterações genéticas do Huntington foram referenciadas nos estudos de Gil-Mohapel e Rego (2011), referindo-se sobre a transmissão da patologia por intermédio do DNA através da genética, seguindo as regras de hereditariedade Mendeliana, em que, os alelos autossômicos dominantes são transmitidos de geração em geração.

Segundo Pereira (2015), o cromossomo 4 normalmente apresenta de 5 a 35 repetições em seus alelos, pessoas com sequências de CAG entre 28 e 35 apresentam o alelo normal com possibilidades para possíveis mutações, o que representa uma faixa de classificação intermediária com potencial expansão nas próximas gerações. As pessoas com Huntington expressam as repetições desordenadas de 36 a 100 alelos de CAG, exemplificado no quadro -1.

A sequência repetitiva desses alelos desencadeia a formação de uma proteína chamada Huntingtina (HTT), funcionalmente alterada que provoca a degeneração neuronal observadas nas diversas regiões do sistema nervoso central, apresentando maior evidência em neurônios do núcleo caudado e putâmen dos gânglios da base (PEREIRA, 2015). Segundo Khan et al., (2020 p. 40), a doença de Huntington está ligada a expansões repetidas de citosina, adenina e guanina (CAG) no gene Huntingtin (HTT),

Quadro 1. Classificação do risco de transmissão da doença com base no número de repetições CAG.

\begin{tabular}{|c|c|c|}
\hline $\begin{array}{l}\text { Número de repetições } \\
\text { CAG }\end{array}$ & $\begin{array}{l}\text { Risco de transmissão } \\
\text { para descendência }\end{array}$ & Manifestação da doença \\
\hline$<28$ & Não há transmissão & $\begin{array}{c}\text { Não há manifestação da } \\
\text { doença }\end{array}$ \\
\hline $28-35$ & $\begin{array}{l}\text { Intermediário (no } \\
\text { caso da ocorrência } \\
\text { de mutação que } \\
\text { levam a um aumento } \\
\text { do número de CAGs } \\
\text { na descendência) }\end{array}$ & $\begin{array}{c}\text { Não há manifestação da } \\
\text { doença }\end{array}$ \\
\hline $35-40$ & $\begin{array}{l}\text { Penetrância } \\
\text { incompleta }\end{array}$ & $\begin{array}{c}\text { Possibilidade de vir a } \\
\text { manifestar a doença ou início } \\
\text { mais tardio }\end{array}$ \\
\hline $40-50$ & $\begin{array}{l}\text { Penetrância } \\
\text { completa }\end{array}$ & $\begin{array}{l}\text { A doença é manifestada na } \\
\text { idade adulta }\end{array}$ \\
\hline$>50$ & $\begin{array}{l}\text { Penetrância } \\
\text { completa }\end{array}$ & $\begin{array}{c}\text { A doença é manifestada } \\
\text { precocemente (durante o } \\
\text { período juvenil) }\end{array}$ \\
\hline
\end{tabular}


que dão origem a mutações que levam ao alongamento do trato da poliglutamina e à formação do produto da proteína HTT.

O conhecimento atual sobre os mecanismos fisiopatológicos relevantes subjacentes à mutação causal do Huntington permanece pouco conhecido. Sabe-se que a presença da proteína HTT mutante é responsável por uma variedade de processos normais que acabam resultando na morte de neurônios. A proteína HTT é uma grande molécula de proteína que consiste em muitas unidades de repetição helicoidal (KHAN et al., 2020).

Esse processo fisiopatológico biomolecular na $\mathrm{DH}$ ocorre por intermédio de disfunção metabólica, apoptose, danos oxidativo ao DNA, agregação proteica, e células neuronais danificadas, a suplementação de alguns componentes dietéticos podem oferecer certo efeito neuroprotetor, tais como a creatina, ácidos graxos poli-insaturados, co-fatores mitocondriais (Co Q10), e L-arginina (ROOS, 2010).

O diagnóstico da doença de huntington nem sempre é fácil e pode ser ponderado em 4 situações: paciente com dados clínicos compatíveis e antecedentes familiares confirmados; paciente assintomático, porém, com antecedentes familiares; quadro clínico típico e ausência de história familiar e por último, clinica atípica sem antecedentes familiares (DINIS, 2015).

Embora sejam os sintomas cognitivos e psiquiátricos fonte de inquietação para o paciente e familiares, são as manifestações motoras a principal razão pela qual o doente procura ajuda médica (GONÇALVES, 2013). Os transtornos motores em conjunto com as alterações psiquiátricas e a disfunção cognitiva os sintomas mais marcantes no quadro clinico da fisiopatologia Huntington (BARRETO, 2009). Os sintomas motores da doença são facilmente identificáveis, representados pelos movimentos involuntários desordenados (RUIZ-ESPIGA, 2010). Nos estudos de Januário (2011) define-se a coreia como movimento involuntário, ondulante, inesperado de amplitude e velocidade variável que surge ao acaso em diversos grupos musculares, mas especialmente na face e tronco.

Peculiares são também os movimentos de extensão que envolvem a musculatura dorso lombar, conferindo uma postura característica ao doente e dificultando a marcha (JANUÁRIO, 2011). A deambulação torna-se instável com movimentos repentinos e vacilantes, a longitude do passo é irregular e apresenta um aumento da base de sustentação que podem originar quedas (HEDREEN; ROOS, 2012).

Com a evolução da doença aparecem também um extenso conjunto de discinesias, tanto hiper como hipocinesias, frequentemente associadas (RUIZ-ESPIGA, 2010) Estas últimas em conjunto com bradicinesias e acinesia, são mais evidentes na fase infantil/juvenil e nos estádios mais avançados da doença (HEDREEN; ROOS, 2012), também se pode observar com frequência distonia, mioclonia, tics (LÓPEZ; BURGUERA, 2010).

Os cuidados com pacientes com doença de huntington denotam grandes desafios, a falta de tratamento definitivo pode ser frustrante, mas atenção especial para as mudanças dos sintomas e a boa comunicação entre os profissionais, membros da família e os indivíduos afetados contribuem para uma conduta bem-sucedida da doença (ROSENBLATT, 2006).

Segundo Rafelsbauer (2009), a progressão da DH pode dividir-se em 3 estágios, no primário a pessoa tem o diagnóstico da doença, realiza as atividades domésticas com dificuldades; avançando ao estágio intermédio o paciente demonstra dificuldades acentuadas para desenvolver atividades remuneradas, responsabilidades domésticas, precisando de orientação e ajuda para temas financeiros; por último, estágio avançado em que o acometido torna-se dependente para as suas atividades diárias, apresentando necessidades de cuidados específicos por parte de seus familiares e enfermagem.

Conforme relatado nos estudos de Caires et al. (2018), esta enfermidade apresenta um grande declínio nutricional, como a perda de peso progressiva levando o paciente a desnutrição severa, causando o possível agravamento de seu estado clinico, outro fator preocupante relaciona-se à broncoaspiração causado pelos disfagia e dificuldades na deglutição podendo ocorrer um desvio de líquidos para os pulmões causando os frequentes sufocamentos e, resultando no desenvolvimento de pneumonia.

As necessidades do paciente e da família dependem da natureza e gravidade das alterações físicas, cognitivas e psicológicas experimentadas pelo paciente. O plano de tratamento aborda estratégias para tratar os sintomas, como Coréia, deglutição, limitações na deambulação. Acompanhamento com o profissional fonoaudiólogo é indicado também para auxiliar nas estratégias de comunicação (MATOS et al., 2011).

Segundo a tese descrita por Dinis (2015, p. 29)

A abordagem reabilitadora nestes doentes é muito importante. A nível motor as mobilizações, treino de equilíbrio, reeducação da marcha e prevenção de quedas são essenciais para prevenir o declínio motor e assim contribuir para a manutenção das capacidades e autonomia do paciente. Na perspectiva cognitiva, a estimulação diária destes pacientes em conjunto com uma eficaz terapia ocupacional de modo a manter as mentas 
ativas e socialmente integradas, é uma das melhores estratégias clínicas para conservação das capacidades funcionais $e$ cognitivas do doente de Huntington. A terapia da fala é outra das valências de importante intervenção, tanto a nível da linguagem, quando o paciente começa com disartria tornando o discurso menos inteligível, como da alimentação, com a consciencialização do processo de mastigação e deglutição para evitar episódios de engasgamento que podem levar a broncos aspirações.

O tratamento medicamentoso e não-medicamentoso deve ser adaptado respeitando a individualidade de cada organismo, uma vez que os sinais e sintomas diferem entre os pacientes (ROSS, 2010).

$\mathrm{O}$ tratamento medicamentoso para $\mathrm{DH}$ estão baseados na sintomatologia apresentada pelo paciente e nas melhorias da qualidade de vida (FRANK; JANKOVIC, 2010). Geralmente as medicações utilizadas estão voltadas para as indicações psiquiátricas em decorrência da disfunção dos circuitos neuronais que são acometidos (SANJUAN; BATES, 2011), entre eles encontra-se em maior frequência fármacos antidopaminergicos, antagonistas de glutamato e metabólicos energéticos (MESTRE, et al. 2009).

Utilizando como base a escala que regulamenta a avaliação funcional Unified Huntington's Disease Rating Scale (UHDRS), que verifica quatro domínios principais; a função motora, resposta cognitiva, comportamento e capacidade funcional (TUMAS et al., 2004). Cada item possuem uma série de questões que são pontuadas, sendo que uma pontuação elevada indica maior comprometimento do paciente (YOUSSOV et al., 2013).

Os centros responsáveis pelos cuidados aos pacientes com DH, empenham-se em fornecer, o melhor nível de cuidado possível, atestado pelo fato de que a maioria das instituições apresentam equipes multidisciplinares capazes de adaptar as estratégias de tratamento para necessidades individuais do paciente (MACIEL et al., 2013; MARTELLI, 2014).

Quando o assunto é a composição da equipe multidisciplinar com propósito de acrescentar melhorias ao acompanhamento dos pacientes com doença de Huntington, alguns estudos divergem na composição profissional dessa equipe. Segundo Ramos et al. (2018), o apoio de uma equipe multiprofissional é fundamental para a realização da assistência às famílias acometidas pelas Doenças Neurodegenerativas Raras, devendo ser acompanhadas durante todos os ciclos da vida e na inserção desta pessoa nos cuidados paliativos, que deve acontecer o mais precoce possível, com apoio fisioterápico, terapia ocupacional, assistência social e psicológica, visando, sobretudo, o retardamento da progressão dos sintomas (RAMOS, et al., 2018).

Nos estudos de Maciel et al. (2013), suas descrições sobre os cuidados de pacientes com $\mathrm{DH}$ muitas vezes requer o envolvimento de uma equipe multidisciplinar de saúde, que inclui profissionais da área da enfermagem, fisioterapia, fonoaudiólogos, psicólogos, terapeutas ocupacionais e conselheiros genéticos, que têm papéis importantes na melhoria da qualidade de vida, dados similares foram encontrados no estudo de Martelli (2014). Singer (2012) sugere que agregar outros profissionais a equipe multidisciplinar, como por exemplo, neurologistas, psiquiatras, assistentes sociais, nutricionistas, biomédicos, podem surtir resultados positivos ao tratamento, suprindo possíveis déficit apresentados pelos acometidos.

Segundo Baumgart et al. (2015), o fator nutricional apresenta-se como principal aliado na importante manutenção da saúde, podendo amenizar as alterações decorrentes do envelhecimento, das doenças neurológicas, corroborando com a diminuição dos efeitos deletérios.

Um problema recorrente nos pacientes com doença de Huntington é a perda de peso, consequentemente pela redução do alimento ingerido por causa da disfagia, da fatiga e da depressão. Os acometidos pela doença de Huntington podem apresentar uma necessidade calórica elevada, causados pelo excessivo gasto energético devido os movimentos involuntários ou pelo hipermetabolismo, podendo haver outra razão metabólica não totalmente entendida. (ROSENBLATT, 2006; ASSOCIAÇÃO BRASIL HUNTINGTON, 2015).

As determinações nutricionais devem ser calculadas e direcionadas a suprir as necessidades calóricas apresentadas pelos pacientes com hipermetabolismo, adequando a dieta conforme os déficit apresentados. Pacientes com Huntington apresentam dificuldades em se alimentar, dessa forma encorajá-los a comer pode ser uma opção, ainda que mediante a falta de apetite, a fim de obterem as quantidades necessárias de nutrientes e calorias que supram as necessidades energéticas (ASSOCIAÇÃO BRASIL HUNTINGTON, 2015). A prescrição dietética determina o tipo, a quantidade e a frequência da alimentação com base no processo de doença do indivíduo e nas metas de tratamento de doença (MAHAN, 2012).

$A$ avaliação nutricional é exigida como parte do cuidado integral do paciente e utiliza a interpretação conjunta de diversos parâmetros, dentre os quais estão a história nutricional, socioeconômica e clínica, exame físico nutricional, antropometria e dosagem dos parâmetros 
bioquímicos dos pacientes (LIMA; OLIVEIRA, 2016 p.113).

Para a análise antropométrica do estado nutricional em adultos e idosos utiliza-se os parâmetros antropométricos de peso, estatura, circunferência cintura (CC) como indicador de adiposidade profunda, e circunferência do braço (CB), essa circunferência é muito utilizada para diagnosticar alterações da massa muscular corporal total e, assim, o estado nutricional protéico (CUPPARI et al., 2014).

Utilizando índice de massa corporal para análise das condições nutricionais, calculando a partir da divisão da massa corporal em quilogramas pela estatura em metro elevado ao quadrado obtendo o resultado em $\mathrm{kg} / \mathrm{m}^{2}$, avaliando por meio dos pontos de corte recomendados pela Organização Mundial de Saúde (OMS), para avaliação da população adulta e idosa como descritos no [quadro-2] (BRASIL, 2011).

Segundo a Associação Brasil Huntington (2015), pessoas com Huntington têm ótimo apetite. A frustração está na dificuldade de engolir comida suficiente de modo rápido, sem engasgar, ou até mesmo de direcionar o alimento a boca, devido os movimentos involuntários, agravando os problemas psicossociais relacionados a comida e nutrição.

No estudo Mercês et al. (2018, p. 06), houve uma descrição do estado da paciente com Huntington.

(...) foi descrito uma dietoterapia especifica que contemplasse as necessidades da paciente, evoluindo do quadro de desnutrição severa para o estado de eutrofia, sempre tomando precauções sobre a oferta alimentar para que a paciente não sofresse uma hiperalimentação devido seu estado nutricional, a dieta ofertada foi hipercalórica e hiperproteica com uso de suplementos nutricionais, adição de gorduras boas (azeite de oliva, abacate, leguminosas, pasta de amendoim) nas principais refeições e nas refeições intermediárias, preocupando-se em ofertar alimentos macios e de fácil absorção, em consistência pastosas ou brandas para facilitar a deglutição da mesma e evitar bronco aspiração.

Em pacientes com hipermetabolismo que apresente gasto energético elevado, avalia-se a necessidade de agregar suplementação intercalando as refeições principais. Segundo Mercês et al. (2018), a paciente foi suplementada com fórmula nutricional, conforme as necessidades apresentadas pela participante da pesquisa, contendo densidade calórica de $1.5 \mathrm{kcal} / \mathrm{ml}$ e $422 \mathrm{kcal} / 100 \mathrm{~g}$, o qual atribuiu resultados positivos.

O acompanhamento nutricional pode corroborar desde a seleção dos alimentos até os suplementos mais apropriados para manutenção das necessidades calóricas do paciente. A recuperação do peso corpóreo, algumas vezes, resulta na melhora da precaução, da sensibilidade e muitas vezes parece reduzir a coréia (ASSOCIAÇÃO BRASIL HUNTINGTON, 2015).

Segundo Mercês et al. (2018, p. 09) "O sucesso ou fracasso da intervenção nutricional depende de muitos fatores", a saúde do paciente, a abordagem, o metabolismo do indivíduo ou até mesmo a aceitação da dietoterapia, portanto, a pesquisa demonstrou através de dados relativos sobre a importância da intervenção nutricional no acompanhamento dos pacientes com Huntington, evoluindo seu estado de desnutrição severa para eutrofia.

\section{CONSIDERAÇÕES FINAIS}

O Huntington é uma doença neurodegenerativa progressiva, sem cura, que apresenta em sua sintomatologia declínio cognitivos, desordens no sistema nervoso central, morte neuronal que corrobora com desiquilíbrios nas

Quadro 2. Dados da Organização Mundial da Saúde: Classificação IMC.

\begin{tabular}{|c|c|}
\hline $\begin{array}{c}\text { DIAGNÓSTICO NUTRICIONAL } \\
\text { Magreza severa }\end{array}$ & IMC $=\mathbf{K G} / \mathbf{M}^{2}$ \\
\hline Magreza moderada & $<16,0$ \\
\hline Magreza leve & $>16,0 \mathrm{a}<17,0$ \\
\hline Eutrofia & $>17,0 \mathrm{a}<18,5$ \\
\hline Sobrepeso grau I & $>25,0 \mathrm{a}<30,0$ \\
\hline Sobrepeso grau II & $>30,0 \mathrm{a}<40,0$ \\
\hline Sobrepeso grau III & $>40,0$ \\
\hline
\end{tabular}


funções motoras e déficit nutricionais. Os acometidos apresentam necessidades de acompanhamento domiciliar ou hospitalar, o que seria de grande importância que esse acompanhamento fosse feito por uma equipe multidisciplinar da saúde composta por diversos profissionais como fonoaudiólogo, psicólogo, nutricionista, fisioterapeuta, médico, enfermagem, terapia ocupacional, biomédico, odontólogo.

Enfatizando a relevância do acompanhamento nutricional ao paciente com doença de Huntington, tendo como base o estado de desnutrição severa que a grande maioria dos pacientes apresentam, a disfagia, o hipermetabolismo, o elevado gasto energético total respeitando os princípios básicos da nutrição. A dietoterapia voltada ao paciente com doença de Huntington deve ser contemplada com "adequação" os alimentos devem ser apropriados respeitando as diferentes fases da doença, "qualidade" dieta deve conter variedades de alimentos que satisfaçam as necessidades do corpo, "quantidade" deve ser essencial para não deixar o paciente desnutrir, com cuidado para não hiperalimentar, "harmonia" equilíbrio entre os alimentos em relação a quantidade, qualidade, consistência, e variedade de alimentos que supram as necessidades de micros e macros nutrientes.

Fica, portanto, explicitada a importância do nutricionista junto a equipe multidisciplinar da saúde para o acompanhamento dos pacientes com Huntington, podendo orquestrar ações de saúde com apoio social e dietéticos aos pacientes e cuidadores familiares.

\section{REFERÊNCIAS}

ANDRÉ, V. M.; CEPEDA, C.; LEVINE, M. S.; Dopamina and Glutamate in Huntington's Disease: A Balancing Act. CNS neuroscience \& therapeutics. England, v. 16, n. 3, p. 163-178, Abr. 2011.

ASSOCIAÇÃO BRASIL HUNTINGTON. Nutrição e Doença de Huntington: Guia familiar.2015 [Acesso em 29 de jul. de 2019] Disponível em: http://abh.org.br/wpcontent/uploads/biblioteca/Nutricao/nutricao e dh hds a sem revisao tecnica.pdf

BAUMGART, M.; SNYDER, H.M.; FAZIO, S.; KIM, H. et al. Summry of the evidence on modifiable risk factors for cognitive decline and dementia: A population-based perspective. Alzheimer's Dement. Jun. 11(6):71826.2015

BARRETO, R.D. Características da Disfunção cognitiva na Doença de Huntington. Coimbra: Universidades de Coimbra, 2009.

BRASIL. Ministério da Saúde. Secretaria de Atenção à Saúde.
Departamento de Atenção Básica. Orientações para a coleta e análise de dados antropométricos em serviços de saúde: Norma Técnica do Sistema de Vigilância Alimentar e Nutricional - SISVAN / Ministério da Saúde, Secretaria de Atenção à Saúde, Departamento de Atenção Básica. - Brasília: Ministério da Saúde, 2011. 76 p.: il. - (Série G. Estatística e Informação em Saúde).

CAIRES, A. S.; MARECO, L. A.; MERCÊS, Z. C.; SANTOS, M. R. N. S.; SIMÕES, D. V. A importância do nutricionista na equipe multidisciplinar no tratamento e reabilitação dos portadores de Huntington. Aberto Journal of Physiology and Pathophysiology, 2018, 1: 2 DOI: 10.28933 / ojpp2018-06-1802.

CUPPARI, L. et al. Guia de nutrição: clínica no adulto / coordenação deste guia Lilian Cuppari. - 3. ed. -- Barueri, SP: Manole, 2014. -- (Série guias de medicina ambulatorial e hospitalar / editor Nestor Schor).

CARVALHO, K.G. A mortalidade pela doença de huntington no brasil no período de 1996 a 2015. Faculdade de Ciências da Educação e da Saúde. Brasília, 2018.

COSTA, F. A. G. et al. Doença de Huntington: uma revisão bibliográfica. In: II Congresso Nacional de Pesquisa em Ciências Sociais Aplicadas - II CONAPE, 2013. Disponível em: <www.unioeste.br/eventos/conape>. Acesso em: 05 de julho de 2019.

COTRIM, N. A.; ALCÂNTARA, P. F. P. Huntington: uma análise através da história. Simpósio TCC e Seminário de IC, 2016.

CUNHA, H. T.; LOPES, F. B. Apenas mais um acidente de trabalho? Relato de um caso clínico de coreia de Huntington. Rev. Port. Med. Geral. Fam. v.32 n.2. p.110116. Lisboa, 2016.

DINIS, A. I. M. C. Qualidade de Vida dos Cuidadores Informais de Doentes de Huntington. Tese de Mestrado em Enfermagem de Reabilitação/ Alexandra Isabel Marques da Costa Dinis.2015.

EVANS, S. J.W. et al. Prevalence of Adult Huntington's Disease in the UK Based on Diagnoses Recorded in General Practice Records. Journal of Neurology, Neurosurgery, and Psychiatry. London. v. 84.n. 10, p.1156-1160. Out. 2013.

FERRAZ, C.C.B. et al. Sistematização da assistência de enfermagem ao paciente com doença de Huntington: relato de experiência. Revista de enfermagem da Universidade Federal de Pernambuco. Recife, v. 7, n.7. p. 4796-800. Jul. 2013.

FRANK, J. Advances in the Pharmacological Management of Huntington's disease. Drugs. Auckland.v.19, n. 3 e 4, p. 561-571. Mar.2010.

GIL-MOHAPEL, J. M.; REGO, A. C. Doença de Huntington: uma revisão dos aspectos fisiopatológicos. Rev. 
Neurocienc. $2011 ; 19(4): 724-734$.

GITLER, A. D.; DHILLON, P.; SHORTER, J. Neurodegenerative Disease: Models, Mechanisms and a new hope. Dis Model Mech. V. 10, N. 5. P. 499-502, 2017. Disponível em $<$

https://www.ncbi.nlm.nih.gov/pmc/articles/PMC545117 7/>, acesso em: 06 de julho 2019.

GONÇALVES, N.F.C. Doença de Huntington: uma revisão. Dissertação para obtenção do Grau de Mestre em Medicina. Covilhã- Portugal, 2013.

HEDREEN, J. C.; ROOS, R. A. Enfermedad de Huntington. In D. Dickson, \& R. Weller, Neurodegeneración: Patología molecular de la demencia y los trastornos del movimiento (pp. 258-268). Madrid: Panamericana, 2012.
HEEMELS,
M.
T. "Doenças

neurodegenerativas". Nature. vol. 539, não. 7628, 2016, p. 179. Centro de Referência de Saúde Academic.

HERISHANU, Y.O.; PARVARI, R.; POLLACK, Y. et al. Journal of the Neurological Sciences Huntington disease in subjects from an Israeli Karaite community carrying alleles of intermediate and expanded CAG repeats in the HTT gene: Huntington disease or phenocopy. Journal of the Neurological Sciences, v. 277, n. 1-2, p. 143-146, 2009.

JANUÁRIO, C. Doença de Huntington, Onde estamos agora? Coimbra: Universidades de Coimbra, 2011.

KHAN, H.; ULLAH, H.; TUNDIS, R.; BELWAL, T.; DEVKOTA, H. P.; DAGLIA, M.; CETIN, Z.; SAYGILI, E. I.; CAMPOS, M. G.; CAPANOGLU, E.; DU, M.; DAR, P.; XIAO, J.; Dietary Flavonoids in the Management of Huntington's Disease: Mechanism and Clinical Perspective. eFood. vol. 1(1); February 2020, pp. 38-52.

LIMA, A. C. G.; OLIVEIRA, M. C. Avaliação nutricional de pacientes neurológicos em UTI. Rev Bras Nutr Clin 2016; $31(2): 112-7$

LÓPEZ, L. J. V.; BURGUERA, J. A. H. Enfermedad de Huntington: Claves y respuestas para un desafio singular. Madrid: Panamericana, 2010.

MACEDO, V. F. B.; PRADO, M. R.; BARBOZA, A. M. M.; BARBOZA, H. M. M. Doença de Huntington juvenil: revisão de literatura. GEPNEWS, Maceió, a.3, v.2, n.2, p.16-22, abr./jun. 2019.

MACIEL, R.O.H.; CARDOSO, F. E. C.; CHANA-CUEVAS, P.; COSENTINO, C.; FERNANDEZ, W.; RIEDER, C. R. M. et al. Care of patients with Huntington's disease in South America: a survey. Arq. Neuro-Psiquiatr. 2013; 71:368370.

MAHAN, L.K.; ESCOTT-STUMPS.; RAYMOND, J. L. KRAUSE: Alimentos, nutrição e dietoterapia; [tradução Cláudia Coana, et al.]. -Rio de Janeiro: Elsevier, 2012.

MATOS, D.C.S.; PERPÉTUO, A. M. A.; RODRIGUÊS, C. F.A. Atuação fisioterapêutica na doença de Huntington.
Programa de pós graduação Atividade Física Adaptada e saúde. 2011

MARTELLI, A. Aspectos clínicos e fisiopatológicos da doença de Huntington. Rev. Arch Health Invest. FMG. 2014.

MENDES, L. P.; CYSNEIROS, R. M.; ABREU, E. S. de; CHAUD, D, M. A. Avaliação do estado nutricional e consumo alimentar em pacientes com doença de Alzheimer. Revista da Universidade Vale do Rio Verde, Três Corações, v. 14, n. 2, p. 502-515, ago./dez. 2016.

MERCÊS, Z. C. das; MUNIZ, S. L.; CAIRES, A. S.; SIMÕES, D.L.V. Intervenção nutricional no tratamento do paciente com doença de huntington: estudo de caso. Biblioteca Faculdade Estácio Macapá. Macapá -AP, 2018.

MESTRE, T.; FERREIRA, J. et al. Therapeutic interventions for symptomatic treatment in Huntington's disease; Cochrane Database of Systematic Reviews. Oxford .2009, Issue 3. Art. No.: CD006456.

NANCE, M.; PAULSEN, J. S.; ROSENBLATT, A.; WHEELOCK, V. A physician's guide to the Management of Huntington's disease. Third Edition. Canada: Huntington's Disease Society of Canada, 2011.

PAULA, C. Z. Novos alvos farmacológicos para as doenças neurodegenerativas: Esclerose Lateral Amiotrófica e Doença de Huntington. 2015, 81 f. TCC (Pós-graduação em Farmacologia). Universidade Federal de Minas Gerais, Belo Horizonte.

PEREIRA, L. P. Estudo molecular da doença de huntington e correlações com as manifestações clínicas/Lorraine Poltronieri Pereira- 2015.

RAFFELSBAUER, D. Enfermedad de Huntington, Preguntas y respuestas. European Huntington's DiseaseNetwork. Alemanha, 2009.

RAMOS, N.O.; CANIZARES, V. S. A.; FRANÇA, A. K.; CAMPELO, T. N. C.; CEDARO, J. J. Doença neurodegenerativa rara: caracterização dos portadores de doença de huntington e ataxia espinocerebelar na Amazônia ocidental, Brasil. Rev. Saúde Públ. Paraná. 2018 Dez.;1(2):63-74

ROOS, R. A. Huntington's disease: a Clinical review. Orphanet J Rare Dis. 2010; 5:40.

RUIZ-ESPIGA, P. J. Manifestaciones motoras. In L. J. López del Val, \& J. A. Hernández, Enfermedad de Huntington: claves y respuestas para un desafío singular (pp. 25-30). Madrid: Panamericana, 2010.

SANJUAN, B. The importance of integrating basic and clinical research toward the development of new therapies for Huntington disease. The Journal of clinical investigation. New Haven. v.1, n. 2. p. 476-483. Fev. 2011.

SILVA, T. F. Níveis de mindfulness e injustiça percebida em indivíduos com Doença de Huntington e familiares/ Tânia Fernandes Silva - São Paulo, 2017.

SINGER, C. Comprehensive treatment of Huntington disease 
and other choreic disorders. Cleveland Clinic Journal of Medicine, v. 79, n. 2 (suppl.), p. S30-S34, 2012

SPLITZ, M. Doença de Huntington e outras coreias. Rev. Hosp. Univ. Pedro Ernesto, UERJ. 2010.

TUMAS, V. et al. Internal consistency of a Brazilian version of the unified Huntington's disease rating scale. Arquivos de Neuro-Psiquiatria. São Paulo. v. 62, n. 4, p. 977-982, dez. 2004.

YOUSSOV, K. et al. Unified Huntington's Disease Rating Scale for Advanced Patients: Validation and Follow-up Study. Movement disorders: official journal of the Movement Disorder Society. New York. v. 1, n.1, p.1717-1723. Out. 2013.

$\mathrm{XU}$, M.; ZHI-YING, W. Huntington Disease in Asia. Chinese Medical Journal. Beijing. v. 128, n.13, p. 1815-1819.jul. 2015.

Submissão: $15 / 03 / 2020$

Aprovado para publicação: 10/07/2020 